\title{
INTERNAÇÕES HOSPITALARES DE IDOSOS RESIDENTES DE TRES MUNICIPIOS COM DIFERENTES RENDAS DOMICILIARES PER CAPITA
}

\author{
Simone Stochero Kummer ${ }^{1}$ \\ Irenio Gomes² \\ Claus Dieter Stobäus ${ }^{3}$
}

resumo

Grande parte dos recursos públicos destinados à saúde no Brasil refere-se a internações hospitalares pelo Sistema Único de Saúde (SUS), especialmente com idosos, que são mais suscetíveis a ter doenças. Este estudo teve como objetivo comparar custos de internações hospitalares de idosos de três diferentes municípios (Alvorada, Ivoti e Porto Alegre; com baixa, média e alta renda, respectivamente),

1 Graduada em Enfermagem. Mestre em Gerontologia Biomédica. Doutoranda em Gerontologia Biomédica pelo Instituto de Geriatria e Gerontologia da Pontifícia Universidade Católica do Rio Grande do Sul (PUCRS). E-mail: simone1974@gmail.com.

2 Graduado em Medicina. Doutor em Medicina e Saúde. Professor do Programa de Pós-Graduação Gerontologia Biomédica da Pontifícia Universidade Católica do Rio Grande do Sul (PUCRS). E-mail: irenio.filho@pucrs.br.

3 Graduado em Medicina. Pós-doutor em Psicologia - Universidad Autónoma de Madrid, Espanha. Professor Titular na Faculdade de Educação e do Programa de Pós-Graduação em Educação e em Gerontologia Biomédica da Pontifícia Universidade Católica do Rio Grande do Sul (PUCRS). E-mail: stobaus@pucrs.br. 
extraídos do banco de dados do SHH/SUS - DATASUS. Dados do DATASUS, tabulados via ferramenta TABWIN, foram planilhados em tabela Excel, para comparar totais de custos, frequências por patologia, identificando os 10 maiores aportes de recursos por internações de idosos por meio dos códigos da Classificação Internacional de Doenças - CID, durante o ano de 2011, ressaltando os dois maiores. Em 2011, verificou-se que 42,24\% dos custos hospitalares com idosos de Alvorada estiveram distribuídos entre cinco grupos de códigos CID: I, J, K (1), A (1) e N (1), numa frequência de internações equivalente a 33\%; 62,41\% dos custos hospitalares com idosos de Ivoti estiveram em cinco grupos de códigos CID: I, J, M (2), S (1) e C (1), numa frequência de internações equivalente a 45,22\%; e 40,39\% dos custos hospitalares com idosos de Porto Alegre estiveram em três grupos de códigos CID: I, J e A (1), numa frequência de 28,77\% do total das internações de idosos. Existe elevada frequência dos códigos I - doenças do aparelho circulatório e J - doenças do aparelho respiratório nos três municípios, representando internações mais custosas, identificando perfil de similaridade entre os municípios, podendo-se pressupor deficiências na Atenção Básica a esses idosos.

palavras-chave

Idosos. Internações Hospitalares. Custos. Serviços de Saúde. Renda.

\section{Introdução}

Abordar custos de internações hospitalares de idosos pressupõe a percepção e análise da realidade e de possíveis melhorias a serem incrementadas pela administração pública, em prol da adequada utilização dos recursos. $\mathrm{O}$ Departamento de Informações do Ministério da Saúde utiliza a Classificação Internacional de Doenças (CID) como critério de classificação de doenças prevalentes nas internações hospitalares, considerando esta uma forma de responder à necessidade de conhecer as causas de morte e para identificar situações de morbidade geradoras das internações (DI NUBILA; BUCHALLA, 2008). Diante da identificação de padrões de utilização dos recursos para o custeio de internações de idosos de diferentes municípios, com diferentes realidades, torna-se relevante discutir as responsabilidades da gestão pública em planejar o sistema, para dividir os recursos de modo equânime.

O material acerca de internações em todo o Rio Grande do Sul pelo SUS consta no site do DATASUS (BRASIL, 2013b), em arquivos do Sistema 
de Informações Hospitalares (SIHSUS). São dados públicos, os quais podem ser visualizados por intermédio do Tab para Windows/TABWIN (BRASIL, 2013a), que permite a discriminação específica de dados selecionados, tais como: município de residência do paciente internado, CID relacionado ao procedimento principal da internação, sua faixa etária, valor total de custos da internação, frequência das internações, entre outros.

Kummer (2014) extraiu os dados de CID que englobaram os maiores custos hospitalares (valor total) e que apresentaram maior frequência, dentre as internações hospitalares de idosos no ano de 2011. Outros trabalhos, incluindo os de Di Nubila e Buchalla (2008), Mafra (2010) e Silva (2009), também realizaram este tipo de pesquisa com dados de CID, porém utilizando outros tipos de abordagem e análise.

O SUS tem por princípios a universalidade do acesso, a integralidade e a igualdade da assistência à saúde, buscando a promoção, proteção e recuperação da saúde (BRASIL, 1990), bem como o Estatuto do Idoso (2003). Dentre os princípios do Sistema, ainda estão incluídos a descentralização político-administrativa, a regionalização e hierarquização da rede de serviços, de modo a exigir um financiamento tripartite, entre as três esferas de governo: municipal, estadual e federal.

Esta pesquisa aprofunda discussões relacionadas a dados de custos com internações hospitalares, que são apenas uma parcela do que é considerado custo efetivo em Saúde Pública. Paralelamente, é importante lembrar de custos que não estão inclusos nos valores das internações e que fazem parte da assistência global, quais sejam: investimentos em atenção básica, vigilância em saúde, assistência farmacêutica, entre outros, além do custo do próprio paciente, considerando o particular ou aquele que acessa o sistema de saúde privado (Saúde Suplementar). A utilização do termo custo, de acordo com Ferreira (1993), refere-se à quantia que uma coisa custou ou, conforme Michaelis (2009), ao preço com que se compra uma coisa, valor em dinheiro.

No referencial utilizado como base para essa pesquisa, foram verificadas Leis, Portarias e Relatórios referentes às normativas do Sistema de Saúde do Brasil, tanto no âmbito municipal como nos estadual e federal. O presente trabalho teve por objetivo comparar custos de internações hospitalares de idosos de três diferentes municípios (Alvorada, Ivoti e Porto Alegre; com baixa, média e alta renda, respectivamente), extraídos do banco de dados do SIH/ SUS - DATASUS (BRASIL, 2013b). 
Este estudo é do tipo levantamento, transversal, retrospectivo (GIL, 2002; PINTO; BASTOS, 2007) realizado por intermédio da coleta de dados do site do Departamento de Informática do Sistema Único de Saúde do Brasil DATASUS, sobre todas as internações hospitalares de idosos residentes em três municípios do estado do Rio Grande do Sul, Porto Alegre, Ivoti e Alvorada, que foram intencionalmente selecionados por ordem de renda média domiciliar per capita no ano de 2010, dentre os municípios que pertenciam à ${ }^{1}{ }^{a}$ Coordenadoria Regional de Saúde - CRS. A adoção deste molde metodológico deve-se à relevância em termos de Epidemiologia, pelo objetivo de destacar a prevalência das doenças desencadeadoras das internações de idosos, geradoras dos maiores custos, ao longo do ano de 2011.

O estudo considerou como parâmetro de identificação dos motivos geradores das internações, a Classificação Internacional de Doenças (CID), traçando como desfecho para essa análise os dez códigos da CID que, quando foram classificados no procedimento principal no laudo da $\mathrm{AIH}$, em 2011, originaram a maior despesa em internações de idosos dos três municípios em questão. Foi utilizada a ferramenta Tab para Windows/TABWIN (BRASIL, 2009; 2010; 2011; 2013a), tabulador de dados, acessível na internet, no site do DATASUS, para processar as informações encontradas nos arquivos do Sistema de Informações Hospitalares do Sistema Único de Saúde (SIH/SUS) (BRASIL, 2013b), buscando os seguintes parâmetros para a análise das internações hospitalares de cada um dos municípios em estudo:

- Valor total: foi selecionado o "valor total" de cada uma das internações ocorridas com idosos munícipes dos três municípios, separadamente, no ano de 2011. O valor total refere-se ao custo integral da internação, desde as diárias hospitalares, tanto de leito comum como em unidade de tratamento intensivo (UTI), medicamentos, hemodiálise, materiais, exames de apoio ao diagnóstico e tratamento e outros, incluindo os honorários profissionais.

- Frequência: refere-se ao número de internações de residentes dos três municípios em estudo, ocorridas em hospitais do estado do Rio Grande do Sul, durante o ano de 2011.

Foram incluídos todos os dados contidos no SIH/SUS, em AIH de internações de idosos munícipes de Alvorada, Ivoti e Porto Alegre (doravante colocados em ordem alfabética), independentemente de em que lugar tenham ocorrido as internações hospitalares (dentro do estado do Rio Grande do Sul) e foram excluídos do estudo os dados incompletos e inacessíveis. 
Posteriormente, foram criadas planilhas no programa Excel for Windows ${ }^{\circledR}$ para organizar os dados filtrados no TABWIN e para conseguir realizar uma análise comparativa entre os valores totais das internações hospitalares de idosos e frequência em que ocorrem, de acordo com a patologia geradora de cada internação.

Este estudo considera como parâmetro de identificação dos motivos geradores das internações a Classificação Internacional de Doenças (CID) preenchida como código principal nos laudos de solicitações de autorizações de internação hospitalar (AIH), traçando como objeto de análise os 10 códigos da CID que consumiram o maior aporte de recursos financeiros em cada um dos municípios, entre os dias 01 de janeiro e 31 de dezembro o ano de 2011.

A análise estatística, considerando frequências e percentagens, além de somas e divisões, possibilitou um maior entendimento sobre custos com internações hospitalares dos idosos dos municípios pesquisados. Este trabalho aprofunda a discussão sobre a pesquisa realizada por Kummer (2014), analisando os dez códigos da CID que estiveram citados como motivo principal das internações dos idosos residentes de Alvorada, Ivoti e Porto Alegre (municípios da 1. ${ }^{a}$ Coordenadoria Regional de Saúde que apresentavam a menor, média e mais alta renda média domiciliar, respectivamente), vinculados a procedimentos que geraram o maior custo em internações hospitalares, no ano de 2011.

Os dados coletados na pesquisa de Kummer (2014) são de domínio público, não havendo necessidade de autorização para sua utilização para fins éticos, a não ser a explícita descrição da fonte dos mesmos. Ainda assim, a pesquisa foi submetida ao CEP, que a aprovou em 05/10/2012, número CAAE 06897612.9.0000.5336.

\section{Resultados}

Considerando que o trabalho versa sobre os 10 itens da CID vinculados a procedimentos maiores custos em internações, apresentam-se a seguir as Tabelas 1, 2 e 3, que demonstram os dados averiguados nos três municípios em estudo. 
Tabela 1 - Descrição dos dez códigos da CID relacionados a procedimentos geradores e maior custo financeiro em internações de idosos residentes no município de Alvorada, em 2011

\begin{tabular}{l|c|c|c}
\hline \multicolumn{1}{c|}{ Alvorada } & $\begin{array}{c}\text { Custo das } \\
\text { internações }\end{array}$ & $\begin{array}{c}\text { Frequência de } \\
\text { internações }\end{array}$ & $\begin{array}{c}\text { Valor médio } \\
\text { por internação }\end{array}$ \\
\hline A41 Outras septicemias & $296.617,60$ & 82 & $3.617,29$ \\
\hline I20 Angina pectoris & $248.286,40$ & 62 & $4.004,62$ \\
\hline I50 Insuficiência cardíaca & $246.157,81$ & 256 & 961,55 \\
\hline J96 Insuficiência respiratória & $218.506,79$ & 54 & $4.046,42$ \\
\hline I21 Infarto agudo do miocárdio & $205.775,12$ & 71 & $2.898,24$ \\
\hline J18 Pneumonia p/microrganismos não & $187.226,10$ & 241 & 776,87 \\
\hline especificada & $138.748,12$ & 19 & $7.302,53$ \\
\hline I25 Doença isquêmica crônica do coração & $103.329,46$ & 20 & $5.166,47$ \\
\hline K74 Fibrose e cirrose hepática & $99.793,44$ & 64 & $1.559,27$ \\
\hline N18 Insuficiência renal crônica & $80.697,40$ & 92 & 877,15 \\
\hline J44 Outras doenças pulmonares & & &
\end{tabular}

Fonte: Brasil (2013b).

Tabela 2 - Descrição dos dez códigos da CID relacionados a procedimentos geradores de maior custo financeiro em internações de idosos residentes no município de Ivoti, em 2011

\begin{tabular}{l|c|c|c}
\hline \multicolumn{1}{c|}{ Ivoti } & $\begin{array}{c}\text { Custo das } \\
\text { internações }\end{array}$ & $\begin{array}{c}\text { Frequência de } \\
\text { internações }\end{array}$ & $\begin{array}{c}\text { Valor médio } \\
\text { por internação }\end{array}$ \\
\hline I20 Angina pectoris & $48.402,44$ & 6 & $8.067,07$ \\
\hline I50 Insuficiência cardíaca & $41.813,10$ & 45 & 929,18 \\
\hline $\begin{array}{l}\text { J44 Outras doenças pulmonares obstrutivas } \\
\text { crônicas }\end{array}$ & $35.839,45$ & 54 & 663,69 \\
\hline $\begin{array}{l}\text { J18 Pneumonia p/microrganismos não } \\
\text { especificada }\end{array}$ & $32.309,44$ & 48 & 673,11 \\
\hline I45 Outros transtornos de condução & $14.847,85$ & 2 & $7.423,93$ \\
\hline $\begin{array}{l}\text { I44 Bloqueio atrioventricular e do ramo } \\
\text { esquerdo }\end{array}$ & $14.692,59$ & 2 & $7.346,30$ \\
\hline S72 Fratura do fêmur & $14.535,60$ & 10 & $1.453,56$ \\
\hline M48 Outras espondilopatias & $13.585,80$ & 1 & $13.585,80$ \\
\hline M43 Outras dorsopatias deformantes & $9.530,27$ & 1 & $9.530,27$ \\
\hline $\begin{array}{l}\text { C64 Neoplasia maligna do rim exceto } \\
\text { pelve renal }\end{array}$ & $9.394,79$ & 1 & $9.394,79$ \\
\hline
\end{tabular}

Fonte: Brasil (2013b). 
Tabela 3 - Descrição dos dez códigos da CID relacionados a procedimentos geradores de maior custo financeiro em internações de idosos residentes no município de Porto Alegre, em 2011

\begin{tabular}{l|c|c|c}
\hline \multicolumn{1}{c|}{ Porto Alegre } & $\begin{array}{c}\text { Custo das } \\
\text { internações }\end{array}$ & $\begin{array}{c}\text { Frequência de } \\
\text { internações }\end{array}$ & $\begin{array}{c}\text { Valor médio } \\
\text { por internação }\end{array}$ \\
\hline I20 Angina pectoris & $4.337 .023,10$ & 970 & $4.471,16$ \\
\hline I21 Infarto agudo do miocárdio & $2.544 .968,58$ & 594 & $4.284,46$ \\
\hline $\begin{array}{l}\text { J18 Pneumonia por microrganismos } \\
\text { não especificada }\end{array}$ & $2.201 .675,31$ & 1917 & $1.148,50$ \\
\hline I50 Insuficiência cardíaca & $1.863 .180,61$ & 1240 & $1.502,57$ \\
\hline A41 Outras septicemias & $1.539 .261,25$ & 490 & $3.141,35$ \\
\hline I25 Doença isquêmica crônica do coração & $1.389 .932,45$ & 169 & $8.224,45$ \\
\hline J96 Insuficiência respiratória & $1.356 .195,38$ & 336 & $4.036,30$ \\
\hline I44 Bloqueio atrioventricular e do ramo & $971.697,41$ & 159 & $6.111,30$ \\
\hline esquerdo & $967.632,92$ & 833 & $1.161,62$ \\
\hline J44 Outras doenças pulmonares obstrutivas \\
crônicas
\end{tabular}

Fonte: Brasil (2013b).

Conforme os dados levantados na pesquisa, foi possível perceber que, do total de 2.912 (16,57\%) internações realizadas para pacientes idosos residentes de Alvorada, 33\% (961 internações) foram relacionadas a procedimentos vinculados aos dez CID geradores de maior custo hospitalar, integrantes de cinco Grupos Diagnósticos (I, J, A K e N); entre os idosos de Ivoti, pode-se perceber que do total de 376 internações (18,53\%), durante o ano de 2011, 45\% (170 internações) foram relacionadas aos dez CID mais custosos, os quais pertencem a cinco Grupos Diagnósticos (I, J, M, S e C); e, em Porto Alegre do total de 23.621 (11,15\%) internações realizadas para idosos, 29\% (6.974 internações) estiveram relacionadas aos dez CID, integradas em três Grupos Diagnósticos da CID 10 (I, J e A).

Ao considerar os 10 códigos de CID, representativos dos maiores custos hospitalares entre os idosos dos três municípios, identifica-se percentualmente:

- $42,24 \%$ dos custos hospitalares com idosos, de Alvorada, no ano de 2011, esteve distribuído entre cinco grupos de códigos CID: I, J, K (1), A (1) e $\mathrm{N}(1)$, numa frequência de internações equivalente a $33 \%$.

- $62,41 \%$ dos custos hospitalares com idosos de Ivoti, no ano de 2011, esteve distribuído em cinco grupos de códigos CID: I, J, M (2), S (1) e C (1), numa frequência de internações equivalente a $45,22 \%$. 
- $40,39 \%$ dos custos hospitalares com idosos de Porto Alegre, no ano

Tabela 4 - Descrição do custo com procedimentos relacionados aos grupos diagnósticos da $\mathrm{CID}$, frequência e valor médio destinado às internações hospitalares dos idosos munícipes de Alvorada, Ivoti e Porto Alegre

\begin{tabular}{l|c|c|c}
\hline & $\begin{array}{c}\text { Custo das } \\
\text { internações }\end{array}$ & \multicolumn{1}{c}{$\begin{array}{c}\text { Frequência de } \\
\text { internações }\end{array}$} & $\begin{array}{c}\text { Valor médio } \\
\text { internações }\end{array}$ \\
\hline Grupo I & $838.967,45$ & 408 & $2.056,29$ \\
\hline Grupo J & $486.430,29$ & 387 & $1.256,93$ \\
\hline Grupo A & $296.617,60$ & 82 & $3.617,29$ \\
\hline Grupo K & $103.329,46$ & 20 & $5.166,47$ \\
\hline Grupo N & $99.793,44$ & 64 & $1.559,27$ \\
\hline & & \multicolumn{2}{|c|}{ Alvorada } \\
\hline Grupo I & $119.755,98$ & 55 & $2.177,38$ \\
\hline Grupo J & $68.148,89$ & 102 & 668,13 \\
\hline Grupo M & $23.116,07$ & 2 & $11.558,04$ \\
\hline Grupo S & $14.535,60$ & 10 & $1.453,56$ \\
\hline Grupo C & $9.394,79$ & 1 & $9.394,79$ \\
\hline
\end{tabular}

Porto Alegre

\begin{tabular}{l|c|c|c}
\hline Grupo I & $12.026 .860,80$ & 3.218 & $3.737,37$ \\
\hline Grupo J & $4.525 .503,61$ & 3.086 & $1.466,46$ \\
\hline Grupo A & $1.539 .261,25$ & 490 & $3.141,35$ \\
\hline
\end{tabular}

Fonte: Brasil (2013b).

Com os dados apresentados na Tabela 4, é possível visualizar que o Grupo Diagnóstico I - Doenças do Aparelho Circulatório engloba procedimentos com os maiores custos em internações hospitalares de idosos residentes nos municípios em estudo, durante o ano de 2011, demonstrando em Alvorada um 
custo de $\mathrm{R} \$ 838.967,45$, para a cobertura de 408 internações, perfazendo um percentual de $19,42 \%$ do gasto total com internações de idosos, para $14,01 \%$ das internações. Em Ivoti, o custo foi de R $\$ 119.755,98$ para a cobertura de 55 internações, utilizando um percentual de $31,81 \%$ do gasto total com internações de idosos, para 14,6\% do quantitativo total de internações. Em Porto Alegre, o custo foi de $\mathrm{R} \$ 12.026 .860,80$, para a cobertura de 3.218 internações, para um percentual de $26,85 \%$ dos recursos gastos com internações de idosos em todo o ano de 2011, contemplando $13,62 \%$ do quantitativo total das internações.

Ocupando o segundo lugar no ranking de custos e frequência de internações, os procedimentos relacionados aos códigos da CID J - Doenças do Aparelho Respiratório aparecem totalizando um custo de $\mathrm{R} \$ 486.430,29$, para a cobertura de 387 internações, entre os munícipes de Alvorada, utilizando um percentual de $11,26 \%$ dos recursos gastos com internações de idosos em todo o ano de 2011, e contemplando 13,3\% do quantitativo total de internações. Em Ivoti, obteve-se um custo de R $\$ 68.148,89$ para a cobertura de 102 internações, nas quais foi utilizado um percentual de $18,10 \%$ dos recursos gastos com internações de idosos em todo o ano de 2011 para contemplar 27,13\% do quantitativo total de internações. Em Porto Alegre, foi encontrado um custo de $R$ \$ 4.525.503,61 para a cobertura de 3.086 internações, o que representou um percentual de $10,10 \%$ dos recursos gastos com internações de idosos em todo o ano de 2011, contemplando 13,06\% do quantitativo total de internações.

\section{Discussão}

O município de Alvorada contava, em 2011, com 195.673 habitantes, dos quais 17.569 (9,0\%) eram idosos. Nesse município, 12,42\% dos recursos investidos em saúde da população foram destinados ao pagamento dos custos de 2.912 internações de idosos. Dentre os três municípios em estudo, é o que apresentou a menor renda média domiciliar per capita $(\mathrm{R} \$ 587,84)$, bem como menor valor do Produto Interno Bruto (PIB) (R \$ 7.528,20), com um investimento de R $\$ 147,36$ per capita para o custeio de sua atenção básica no período (KUMMER, 2014).

Em Ivoti, a população era de 19.874 habitantes, sendo 2.029 (10,2\%) idosos. Nessa cidade, $7,15 \%$ dos recursos investidos na saúde da população foram destinados ao pagamento dos custos de 376 internações de idosos. O município possuía uma renda média domiciliar per capita de $\mathrm{R} \$$ 1.019,75 e um PIB de $R$ \$ 22.903,15, no ano de 2010, tendo investido $R$ \$262,17 per capita em atenção básica de saúde (KUMMER, 2014). 
E em Porto Alegre, a população total era de 1.409.351 habitantes, com 211.895 (15\%) de idosos. Do total dos recursos de saúde para o município, 4,86\% (23.621 internações) foi destinado para internações de idosos. Dentre os municípios em estudo, foi o que apresentava a maior renda média domiciliar per capita ( $\mathrm{R} \$ 1.722,37)$ e PIB ( $\mathrm{R} \$ 30.524,80)$, no ano de 2010 tendo investido o valor de $R \$ 113,41$ per capita em sua atenção básica (KUMMER, 2014). A ocorrência de menor quantidade de internações entre os idosos munícipes de Porto Alegre pode ser analisada atreladamente a outras informações, como, por exemplo, à sua população que acessa o Sistema de Saúde por meio de planos privados.

No site da Agência Nacional de Saúde Suplementar, foi possível verificar, na população idosa de cada município, no ano de 2011, o percentual de idosos que possuía acesso à saúde por intermédio de planos privados de assistência médica, que é de 8,94\% dos idosos de Alvorada, 15,81\% dos idosos de Ivoti e $43,85 \%$ dos idosos de Porto Alegre (BRASIL, 2011). A análise desses dados possibilitou identificar que o número de internações hospitalares custeadas pelo SUS aos munícipes de Porto Alegre (respeitando a proporcionalidade populacional) apresentou-se em percentuais menores do que entre os munícipes de Alvorada e Ivoti, o que justifica-se também pela possível utilização do sistema privado por esses idosos.

As classificações diagnósticas do Grupo I e do Grupo J, relacionadas aos procedimentos geradores dos maiores custos, estiveram entre as internações de idosos dos três municípios em estudo, embora sem ocupar posições idênticas na hierarquia de despesas financeiras destinadas pelos municípios. Desse modo, o presente estudo permite uma análise detalhada de diagnósticos de maior custo nas internações hospitalares de idosos dos três municípios pesquisados, confirmando dados já abordados em outro estudo (LA FORGIA; COUTTOLENC, 2009), que afirma que as doenças cardiovasculares e respiratórias são as mais prevalentes nas internações hospitalares de idosos e as que respondem pelos maiores custos hospitalares na população idosa, em média $80 \%$.

A análise dos dados publicados pelo Ministério da Saúde permitiu verificar um total de 15 milhões de internações realizadas, em 2010, no Brasil. Destas, a faixa etária de 60 anos ou mais foi responsável por $22 \%$ delas, com uma prevalência de doenças do aparelho circulatório (30\%), seguidas por doenças respiratórias $(21 \%)$ e um incremento de tratamentos de maior complexidade (KANSO et al., 2013).

Mesmo considerando as diferenças apresentadas entre as internações dos idosos de cada um dos três municípios em estudo, os resultados apontam que 
as internações de maior custo (distribuídas entre procedimentos codificados nos Grupos Diagnósticos I, J, A, K, N, M, S e C) respondem entre 40 e $62 \%$ do total de recursos despendidos. Dentre essas internações, os grupos I e J, doenças cardiovasculares e doenças respiratórias foram os mais incidentes (de acordo com as frequências de internações) e as que demandaram maior despesa nos três municípios pesquisados - 72,6 \% em Alvorada, 80 \% em Ivoti e 91,5 $\%$ em Porto Alegre. Em média, 89,6\% dos recursos gastos nessas internações (de todos os 10 CID mais custosos nos três municípios) foram destinados ao custeio dos procedimentos relacionados a estes dois grupos de classificação diagnóstica, novamente recordando os elementos encontrados por La Forgia e Couttolenc (2009).

No entanto, quando avaliado o custo médio por internações, os dados evidenciam que, para um mesmo grupo de classificação diagnóstica, os custos com os procedimentos não são os mesmos. Comparando os custos com procedimentos por internações, com codificação no grupo I (doenças cardiovasculares) como CID principal da AIH, os idosos de Alvorada e Ivoti assemelham-se: em média $\mathrm{R} \$ 2.100,00$ por internação. Já entre os idosos de Porto Alegre, os custos para procedimentos do mesmo grupo são $43 \%$ maiores, considerando as internações individualmente (quase 3.750,00).

Existe a possibilidade de maior ocorrência, entre os idosos munícipes de Porto Alegre, de internações de maior complexidade, geradoras de maiores custos, em razão de maior frequência de comorbidades, agravos associados, como destaca Zaslavsky e Gus (2002), podendo estar possivelmente relacionados ao menor investimento na atenção básica, já comentado por Kummer (2014), com reduzidas ações de caráter preventivo, ou mesmo à baixa adesão ao tratamento. Também ocorre que os idosos são particularmente suscetíveis a infecções nosocomiais, conforme Góis e Veras (2010), portanto consomem mais dos Serviços de Saúde, tendo internações hospitalares mais frequentes, com um tempo de ocupação do leito maior do que em outras faixas etárias.

Considerando a facilidade de acesso à emergência de hospitais de alta complexidade por idosos em Porto Alegre, pode-se comentar sobre uma maior utilização de tecnologias de alto custo, as quais agregam valores às contas hospitalares, partindo do entendimento de que a maior disponibilidade de serviços e tecnologias em saúde parece predispor à maior utilização dos mesmos (KUMMER, 2014).

Comparando o custo das internações hospitalares de mesmos grupos diagnósticos da CID, entre os idosos dos municípios em estudo, Porto Alegre tem os mais elevados custos por internação, o que levanta a hipótese de influências exercidas pela renda média domiciliar per capita, conjuntamente 
com fatores associados a saneamento e nível de escolaridade (CASTRO; TRAVASSOS; CARVALHO, 2002), para modificar o padrão de necessidades na assistência em saúde (KUMMER, 2014).

Ao analisar-se os custos com os procedimentos relacionados aos diagnósticos do grupo J, as internações dos munícipes de Alvorada e Porto Alegre assemelharam-se, com um custo médio de $\mathrm{R} \$ 1.350,00$. Os munícipes de Ivoti apresentaram um custo 50\% menor, em média $\mathrm{R} \$ 670,00$. Diante dos valores coletados, pode-se perceber que o município de Ivoti possui uma situação de renda intermediária, o que não o coloca, necessariamente, numa situação intermediária de saúde/doença. É o município que conta com estrutura hospitalar de pequeno porte, comparativamente aos demais municípios analisados. Sua atenção hospitalar, em parte dos atendimentos de média complexidade e de alta complexidade, fica referenciada aos municípios de Novo Hamburgo, São Leopoldo e Canoas (RIO GRANDE DO SUL, 2010). Como contraponto, salienta-se que foi o que realizou maior investimento per capita na Atenção Básica, durante o ano de 2011 (KUMMER, 2014).

Mesmo dentro de um mesmo grupo, diagnósticos diferentes aparecem para os três municípios, o que pode explicar porque em um mesmo grupo, o custeio é diferente, quando analisado custo médio das internações. Outras variáveis podem determinar diferenças na média do custo das internações, tais como aquelas que se referem a variações de tratamentos e práticas clínicas para um mesmo diagnóstico, tipos de procedimentos utilizados como escolha, case mix (perfil de pacientes, comorbidades), tempo de internação, inexistência de padrões assistenciais, perfil dos profissionais e outros, diz La Forgia e Couttolenc (2009).

De qualquer forma, os resultados apontam que as doenças cardiovasculares e respiratórias devem continuar a receber uma maior atenção, uma vez que os gastos destinados aos seus tratamentos em internações hospitalares geram uma maior necessidade de recursos por parte do sistema público. Entende-se que o acesso a políticas de Atenção Básica em saúde produz menos internações hospitalares, conforme Mafra (2010).

Apesar dos programas criados pelos governos Federal, Estaduais e Municipais, visando a melhor distribuição da renda, trabalho, melhores condições de moradia e de educação, entre outros, identifica-se que a morbidade entre os idosos residentes nos três municípios em estudo está seguindo nos mesmos índices que os padrões nacionais (LA FORGIA; COUTTOLENC, 2009; KANSO et al., 2013; WORLD HEALTH ORGANIZATION, 2005).

A ampliação de ações focadas na Educação em Saúde pode representar a possibilidade de mudança neste quadro, partindo-se de perspectivas dialógicas, 
emancipadoras, participativas e criativas, que contribuam para maior autonomia do usuário, no que diz respeito à sua condição de sujeito de direitos e autor de sua trajetória de saúde e doença (BRASIL, 2007).

Nesta linha de Educação em Saúde, os profissionais também devem encontrar maior autonomia diante da possibilidade de (re)inventar modos de cuidado mais humanizados, compartilhados e integrais, com possibilidades teóricas e metodológicas para transformar as tradicionais práticas de Educação em Saúde em práticas pedagógicas que levem à superação das situações que limitam o viver com o máximo de qualidade de vida (BRASIL, 2007). Esse movimento já foi ressaltado antes por Mosquera e Stobäus (1984).

O estudo permitiu discutir sobre os dados levantados e inferir que, quanto melhor a renda média domiciliar per capita, maiores as possibilidades para a população encontrar condições, entre elas as socioeconômicas e estruturais, que permitam, mais autonomamente, o desenvolvimento de ações indicadas para incremento e manutenção de uma melhor qualidade de vida. Questões como elevados níveis de escolaridade também podem contribuir para a valorização da inclusão destas ações e consequente possibilidade de mudanças no estilo de vida pessoal e coletivamente (KUMMER, 2014).

Paralelamente, o município que possui um maior PIB per capita recebe mais recursos para investir em Educação e em Saúde, o que também pressupõe melhor possibilidade de Promoção de Saúde e prevenção de doença (KUMMER, 2014). Diante de dados como renda e PIB per capita, é possível que os municípios com a menor renda média domiciliar per capita, que desencadeiam a menor autonomia dos indivíduos e dos gestores no gerenciamento de recursos em possíveis melhorias na qualidade de suas vidas, são também os que poderim gerar a menor quantidade de riquezas.

Dentre as limitações desse estudo, é interessante considerar a codifi cação da CID principal referente à internação como um fator que poderia estar "embutido" (no sentido de poder estar desviando) em eventuais "distorções nos dados", pois nem sempre há informação sobre a doença de base ou o registro de uma complicação da mesma, como por exemplo insuficiência cardíaca, especificada ou não como congestiva (LAURENTI; BUCHALLA; CARATIN, 2000).

Sobretudo, é importante enfatizar que, apesar de algumas limitações, os dados disponibilizados pelo SIH/SUS são uma fonte adequada de informações. No entanto, a diversidade de hospitais e serviços que eles recolhem e a qualidade do preenchimento dos dados pelas equipes desses estabelecimentos são aspectos que poderiam permitir a ocorrência de algum erro ou "problemas" no lançamento das faturas hospitalares, podendo estar interferindo nos dados que foram incluídos. 
Para a realização de estudos posteriores, sugere-se pensar na identifi cação dos hospitais em que ocorreram as internações dos pacientes, para fins de compreender mais detalhadamente os atendimentos prestados, relacionando-os aos custos das contas hospitalares. Agregar a informações da CID secundária referente às internações também poderia contribuir para uma mais precisa análise posterior.

\section{Conclusão}

Verificou-se elevada prevalência dos códigos do grupo CID I e J, referentes às doenças do aparelho circulatório e respiratório, entre as internações mais custosas nos municípios investigados, pressupondo que o incremento de ações na Atenção Básica de Saúde da população pode ter intensa relação na mudança destes dados. Considerando que os códigos averiguados representam a utilização dos serviços de média e alta complexidade, englobando essas populações, e que mesmo tendo perfil sócio-econômico diferenciado apresentam padrões similares de adoecimento, conclui-se a necessidade de implementar maior efetividade em Atenção Básica, Promoção em Saúde e medidas de prevenção.

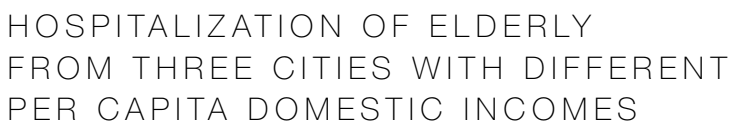

abstract

Large proportion of public resources for health in Brazil are related to hospitalizations by Health Unic System (SUS- Public Health System), especially with the elderly, who are more susceptible to hazards. The objective was compare costs of elderly hospitalization of three different municipalities (Alvorada, Ivoti and Porto Alegre, with low, medium and high income, respectively), taken from the database of SIH/SUS-DATASUS. The method used was the use of official data of DATASUS, tabulated via TABWIN tool, were transferred to Excel table to compare total costs, frequency of disease, identifying the top 10 features contributions by admissions of elderly through the codes of the International Classification of Diseases-ICD, during the year 2011, highlighting the two largest. As results, in 2011, it was found that $42.24 \%$ of elderly hospitalization costs in Alvorada were distributed among five groups of ICD codes: I, J, K (1), A (1) and N 
(1), a frequency admissions equivalent to 33\%; $62.41 \%$ of hospital costs of Ivoti elderly been distributed into five groups of ICD codes: I, J, M (2) S (1) and C (1), a frequency equivalent of 45,22\% of the hospitalizations; and $40.39 \%$ of elderly hospitalizations costs in Porto Alegre were distributed between three groups of ICD codes: I, J and $A(1)$, at a frequency of $28.77 \%$. As conclusion, appears a high frequency of codes I - circulatory diseases and $\mathrm{J}$ - respiratory diseases in the three counties, representing the most costly hospitalizations, identifying profile similarity between the municipalities, which can assume deficiencies in Primary Care.

\section{keywords}

Elderly. Hospital Hospitalizations. Costs. Health Services. Income.

referências

BRASIL. Lei no 8.080, de 19 de setembro de 1990. Dispõe sobre as condições para a promoção, proteção e recuperação da saúde, a organização e funcionamento dos serviços correspondentes e dá outras providências. Diário Oficial da União - Seção I, Brasília, DF, 20 set. 1990. Disponível em: <http://www.planalto.gov.br/ccivil_03/leis/ L8080.htm>. Acesso em: 20 jun. 2015.

Ministério da Saúde. Secretaria de Gestão Estratégica e Participativa. Departamento de Apoio à Gestão Participativa. Caderno de educação popular e saúde. Brasília: Ministério da Saúde, 2007. Disponível em: <http://portal.saude.gov. br/portal/arquivos/pdf/caderno_de_educacao_popular_e_saude.pdf >. Acesso em: 20 jun. 2015.

- Ministério da Saúde. Agência Nacional de Saúde Suplementar. Informações em Saúde Suplementar - ANS Tabnet. Beneficiários por município. Rio de Janeiro: Ministério da Saúde, 2011. Disponível em: <http://www.ans.gov.br/anstabnet/anstabnet/ deftohtm.exe?anstabnet/dados/TABNET_02.DEF>. Acesso em: 15 jun. 2013.

Departamento de Informática do SUS. Download de Programa - TABWIN [Internet]. Brasília: Ministério da Saúde, 2013a. Disponível em: <http://www2.datasus. gov.br/DATASUS/index.php?area=060805\&item=3>. Acesso em: 20 jun. 2015.

Departamento de Informática do SUS. Dados das internações hospitalares no Rio Grande do Sul pelo SUS. Brasília: Ministério da Saúde, 2013b. Disponível em: <http://www2.datasus.gov.br/DATASUS/index.php?area=0901\&item=1\&acao=25>. Acesso em: 20 jun. 2013.

CASTRO, Mônica Silva Monteiro de; TRAVASSOS, Cláudia; CARVALHO, Marília Sá Fatores associados às internações hospitalares no Brasil. Ciência \& Saúde Coletiva, Rio de Janeiro, v. 7, n. 4, p. 795- 811, 2002. Disponível em: <http://www.scielo.br/pdf/ csc/v7n4/14606.pdf>. Acesso em: 12 dez. 2014.

DI NUBILA, Heloísa Brunow Ventura; BUCHALLA, Cássia Maria. O papel das Classificações da OMS - CID e CIF nas definições de deficiência e incapacidade. Revista Brasileira de Epidemiologia, São Paulo, v.11, n. 2, p. 324-335, jun. 2008. Disponível em: <http://www.scielo.br/scielo.php?pid=S1415-790X2008000200014\&script=sci_arttext>. Acesso em: 10 mar. 2013. 
FERREIRA, Aurélio Buarque de Holanda. Minidicionário Aurélio da Língua Portuguesa. 3. ed. Rio de Janeiro: Nova Fronteira, 1993.

GIL, Antonio Carlos. Como elaborar projetos de pesquisa. 4. ed. São Paulo: Atlas, 2002.

GÓIS, Ana Luzia Batista de; VERAS, Renato Peixoto. Informações sobre a morbidade hospitalar em idosos nas internações do Sistema Único de Saúde do Brasil. Ciência \& Saúde Coletiva, Rio de Janeiro, v. 15, n. 6, p. 2859-2869, set. 2010. Disponível em: $<$ http://www.scielo.br/scielo.php?script=sci_arttext\&pid=S1413-81232010000600023>. Acesso em: 20 jul.2014

KANSO, Solange et al. A evitabilidade de óbitos entre idosos em São Paulo, Brasil: análise das principais causas de morte. Cadernos de Saúde Pública, Rio de Janeiro, v. 29, n. 4, p. 735-748, abr. 2013. Disponível em: <http://dx.doi.org/10.1590/S0102311X2013000400011>. Acesso em: 21 jun. 2015.

KUMMER, Simone Stochero. Análise comparativa dos custos de internações hospitalares de idosos, pelo SUS, em municípios com diferentes realidades de renda média domiciliar per capita. 2014. 139 f. Dissertação (Mestrado em Gerontologia Biomédica) - Pontifícia Universidade Católica do Rio Grande do Sul, Porto Alegre, 2014. Disponível em: <http://meriva.pucrs.br:8080/dspace/bitstream/10923/5769/1/000457202-Texto\%2BCompleto-0.pdf>. Acesso em: 10 ago. 2014.

LA FORGIA, Gerard M.; COUTTOLENC, Bernard F. Desempenho hospitalar no Brasil: em busca da excelência. São Paulo: Singular, 2009.

LAURENTI, Ruy; BUCHALLA, Cassia Maria; CARATIN, Carla Vanessa de Sousa. Doença isquêmica do coração. Internações, tempo de permanência e gastos. Brasil, 1993 a 1997. Arquivos Brasileiros de Cardiologia, Rio de Janeiro, v. 74, n. 6, p. 483-487, 2000. Disponível em: <http://publicacoes.cardiol.br/abc/2000/7406/74060001.pdf>. Acesso em: 12 ago. 2013.

MAFRA, Fábio. O Impacto da Atenção Básica em Saúde em Indicadores de Internação Hospitalar no Brasil. 2010. 129 f. Dissertação (Mestrado Profissional em Regulação e Gestão de Negócios) - Faculdade de Economia, Administração, Contabilidade e Ciência da Informação e Documentação (FACE), Centro de Investigação em Economia e Finanças (CIEF) e Centro de Estudos em Regulação de Mercados (CERME), Universidade de Brasília, Brasília, 2010. Disponível em: < http://repositorio.unb.br/bitstream/ 10482/9156/1/2011_F\%c3\%a1bioMafra.pdf >. Acesso em: 12 ago. 2013.

MICHAELIS. Moderno dicionário de língua portuguesa Michaelis. São Paulo: Editora Melhoramentos, 2009. Disponivel em: <http://michaelis.uol.com.br/moderno/portugues/ index.php?lingua=portugues-portugues\&palavra=CUSTO>. Acesso em: 17 jun. 2012.

MOSQUERA, Juan José Mouriño; STOBÄUS, Claus Dieter (Org.). Educação para a Saúde. 2. ed. Porto Alegre: D. C. Luzzatto, 1984.

PINTO, Regina Bueno Ribas; BASTOS, Laudelino Cordeiro. Abordagem das pesquisas em Epidemiologia aplicada à Gerontologia no Brasil. Revista Brasileira de Epidemiologia, Rio de Janeiro, v. 10, n. 3, p. 361- 369, 2007.

RIO GRANDE DO SUL. Secretaria da Saúde do Estado do Rio Grande do Sul [site da gestão 2006-2010]. Departamento de Assistência Hospitalar e Ambulatorial - DAHA. Alta Complexidade em cardiologia, traumatologia, oncologia, neurologia e neurocirurgia. Disponível em: <http://www1.saude.rs.gov.br/wsa/portal/index.jsp?menu=organogra ma\&cod=24077>. Acesso em: 2 jun. 2013

SILVA, Norberto Peçanha. A utilização dos programas TABWIN e TABNET como ferramentas de apoio à disseminação das informações em saúde. 2009. 98 f. Dissertação (Mestrado na Modalidade Profissional em Saúde Pública) - Escola Nacional de Saúde Pública Sérgio Arouca, Fundação Oswaldo Cruz, Rio de Janeiro, 2009. 
WORLD HEALTH ORGANIZATION. Enve/hecimento ativo: uma política de saúde. Tradução: Suzana Gontijo. Brasília: Organização Pan-Americana da Saúde, 2005.

ZASLAVSKY, Cláudio; GUS, Iseu. Idoso. Doença cardíaca e comorbidades. Arquivos Brasileiros de Cardiologia, Rio de Janeiro, v. 79, n. 6, p. 635- 639, 2002. Disponível em: <http://www.scielo.br/scielo.php?script=sci_arttext\&pid=S0066-782X2002001500011>. Acesso em: 12 mar. 2013. 